\title{
Course and Outcome of Bacteremia Due to Staphylococcus aureus: Evaluation of Different Clinical Case Definitions
}

\author{
Stephan Lautenschlager, ${ }^{*}$ Christian Herzog, ${ }^{\dagger}$ \\ and Werner Zimmerli
}

From the Department of Clinical Research. F. Hoffmann-La Roche \&
Co., Lid.; and the Division of Infectious Diseases, Department of
Medicine, University Hospital, Basel, Switzerland

\begin{abstract}
In a retrospective survey of patients hospitalized in the University Hospital of Basel, Switzerland, the course and outcome of 281 cases of true bacteremia due to Staphylococcus aureus over a 7-year period were analyzed. The main purpose was to evaluate different case definitions. In 78\% of cases the source of bacteremia was obvious; vascular access sites $(27 \%)$ and wounds $(10 \%)$ were the most common sources. Metastasizing foci were more common in cases of primary vs. secondary bacteremia $(P<.001)$. The incidence of endocarditis was higher in cases in which no portal of entry was defined $(P<.03)$. The overall mortality rate was high at $34 \%$ partly because of inappropriate initial antibiotic therapy. With the introduction of an infectious disease service at the hospital, the fraction of misjudged results of blood culture diminished 2.5-fold. Among the differently defined cases, the mortality rate was significantly higher for cases of complicated vs. uncomplicated bacteremia $(P<.01)$, for cases of primary vs. secondary bacteremia $(P=.05)$, and for patients with endocarditis or other secondary foci $(P<.001)$. Since only one methicillin-resistant strain was isolated, multiresistant staphylococci were not a problem in the hospital. Different case definitions allowed the detection of patients at increased risk for complications and death. In the treatment of sepsis with no evident focus, initial antimicrobial therapy should include the use of agents with antistaphylococcal activity.
\end{abstract}

Since the introduction of antimicrobial agents, considerable changes have taken place regarding the pattern of bacterial species causing bacteremia. Streptococcus pneumoniae and other streptococci have largely been replaced in this role by staphylococci and gram-negative rods [1-12]. During a 7-year period, 2,746 episodes of bacteremia associated with positive blood cultures were diagnosed at the University Hospital in Basel, Switzerland. In 373 episodes (13.6\%) Staphylococcus aureus was isolated. The purpose of this retrospective study was to analyze the course and outcome of 281 episodes of true bacteremia due to $S$. aureus, with special emphasis on acquisition, clinical severity, nature of infection, and primary focus.

\section{Patients, Definitions, and Methods}

The University Hospital of Basel is a 1,000-bed acute care facility. From 1 January 1980 through 31 December 1986

\footnotetext{
Received 22 May 1992; revised 17 November 1992.

* Present address: Clinic of Dermatology. University Hospital, CH-4031 Basel, Switzerland.

${ }^{\dagger}$ Present address: Department of Public Health, St. Albanvorstadt 12. CH-4006 Basel, Switzerland.

Reprints or correspondence: Dr. W. Zimmerli, Division of Infectious Diseases, Department of Medicine, University Hospital, CH-4031 Basel, Switzerland.
}

Clinical Infectious Diseases 1993;16:567-73

(c) 1993 by The University of Chicago. All rights reserved.

1058-4838/93/1604-0020\$02.00 every episode of bacteremia recorded at the Bacteriology Laboratory was registered for the purposes of this study. The medical records of 373 patients for whom a blood culture was positive for $S$. aureus were retrospectively reviewed. Seventeen patients with polymicrobial bacteremia were excluded. In addition, single positive hemocultures for $57 \mathrm{pa}^{-}$ tients for whom no further culture yielded the same phage type were judged as contaminated (see below). Eight of these patients had a subsequent episode of bacteremia due to another pathogen, 21 patients had postoperative fever with spontaneous resolution, and in 28 cases the clinical course did not suggest infection. Records for 18 patients were not available for the study. The remaining 281 medical charts were analyzed for data regarding primary and secondary foci, clinical signs and symptoms, laboratory findings, the treating physician's opinion in regard to the severity of the infection, antibiotic treatment, complications, follow-up, phage type, and in vitro susceptibility of $S$. aureus. If the presence or absence of a particular finding was not clearly indicated on a chart, the case was excluded from analysis in regard to that finding, a practice that resulted in the use of variable denominators in the data we present herein.

Contamination. The positivity of a single blood culture per set (one aerobic and one anaerobic bottle) was considered indicative of contamination, unless an identical strain of $S$. aureus was additionally cultivated from a specimen from a focus in a patient who had appropriate clinical signs $[13,14]$.

Episode. An episode of bacteremia was defined when the results of the cultures were positive or when results of a fur- 
ther hemoculture were positive later than 1 week after negative results were obtained [15].

Acquisition. Bacteremia was judged to be community acquired (CA) when the positive culture results were obtained at or within 48 hours after admission or when there was evidence of $S$. aureus infection on admission [10, 16-18]. Bacteremia was judged to be hospital acquired (HA) when the positive culture results were obtained $>48$ hours after hospitalization. Episodes of bacteremia secondary to infections of dialysis access sites were considered to be due to HA organisms.

Underlying conditions. Glucocorticosteroid therapy was considered a risk factor if a patient had received the daily equivalent of $\geqslant 25 \mathrm{mg}$ of prednisone for at least 1 week prior to the time the positive blood culture results were obtained [19]. Chronic renal failure was defined as a persistent rise in serum creatinine levels to $\geqslant 180 \mu \mathrm{mol} / \mathrm{L}$ [20]. Diabetes mellitus was a risk factor in cases of insulin dependency or chronic treatment with oral hypoglycemic agents [20]. Previous hospitalization within 30 days of onset of illness was viewed as predisposing the patient to septicemia [21]. Other conditions frequently cited as predisposing factors to infection (table 1) were established as being either present or absent in each episode $[4,5,10,16,20-31]$.

Clinical significance. Criteria for clinical significance were used as proposed by Michel et al. [17]. These criteria were the existence of true bacteremia plus at least three of the following factors: leukocyte count, $\geqslant 10 \times 10^{9} / \mathrm{L}$; temperature, $\geqslant 38.5^{\circ} \mathrm{C}$; heart rate, $\geqslant 100 / \mathrm{min}$; chills; hypotension (systolic blood pressure, $<90 \mathrm{~mm} \mathrm{Hg}$ or a fall of $\geqslant 30 \mathrm{~mm}$ $\mathrm{Hg}$ ); or new oliguria (urine output, $<400 \mathrm{~mL} / 24$ hours).

Complicated bacteremia. Bacteremia was considered to be complicated if a focus of infection was absent or nonremovable [32, 33].

Primary and secondary bacteremia. Bacteremia occurring in the absence of an apparent portal of entry was classified as primary. If a portal of entry was identified, bacteremia was defined as secondary.

Portal of entry and secondary foci. The initial staphylococcal lesion leading to bacteremia was defined as the portal of entry. Other foci were considered as sequelae resulting from seeding of the initial lesion. Primary infection of the respiratory and urinary tracts was diagnosed only when symptoms and signs typically associated with bacterial infections of those systems were present and coincident with appropriate results of culture. The presence of Staphylococcus isolates in urine was considered to be secondary to bacteremia if the phage type of organisms isolated from urine and blood matched. An intravascular catheter was considered as the portal of entry if ( 1 ) there was evidence of inflammation at the catheter insertion site and/or (2) a catheter-tip culture was positive for $S$. aureus and (3) there was no evidence of a source of infection elsewhere [34]. Endocarditis was defined according to Nolan and Beaty [23] and Bayer et al. [35].
Prognosis. Patients were classified as three groups according to prognosis: (1) good prognosis (patients without underlying disease); (2) poor prognosis (patients with underlying surgical or medical disorders of such severity that recovery from their primary disease was unlikely); and (3) intermediate prognosis (patients not qualifying for a good or poor prognosis) [36].

Outcome. Treatment failure was defined as clinical deterioration or persistence of disease activity sufficient to warrant a change in the treatment. When data were not available for at least 1 month after completion of antibiotic therapy, the patient was rated as improved instead of cured.

Treatment. Treatment was classified as either local (including the removal of infectious foci or surgical therapy) or systemic (including the initial antibiotic therapy, started before culture results were available, and the antibiotic therapy used after sensitivity testing). Treatment was rated as appropriate or inappropriate according to well-accepted guidelines [37].

Statistical analysis. Statistical analysis was performed with use of programs of the SAS system (SAS Institute, Cary, NC). The $\chi^{2}$ test was used for proportional values, and the Student's $t$-test, for independent populations. A $P$ value of $\leqslant .05$ was considered significant.

\section{Results}

During the 7-year period investigated, 2,746 episodes of bacteremia were diagnosed in the laboratory. The most frequent isolates were Escherichia coli (22.8\%), Streptococcus species (16.2\%), and coagulase-negative staphylococcus (13.2\%). S. aureus was isolated in 373 cases (13.6\%). All cases of staphylococcal bacteremia were observed in either the surgical or the medical wards, with the exception of 14 cases that were observed in departments dealing with the following specialties: gynecology and obstetrics (4); dermatology (5); ear, nose, and throat disorders (2); paraplegic patients (2); and ophthalmology (1). The majority of patients with CA bacteremia ( $85 \%$ ) were hospitalized in the medical departments. The proportion of bacteremic episodes caused by $S$. aureus remained relatively stable over the study period.

Acquisition. A majority of cases (200 [71\%] of 281) were HA; $80(28 \%)$ were CA, and in one case the mode of acquisition was not ascertainable. Over the years the rate of CA cases of bacteremia varied considerably between $14.6 \%$ (1983) and 38.6\% (1986).

Age and sex. The median age was 60 years, within a range of $1-100$ years (only one patient was younger than 10 years old). The majority of cases occurred in the sixth (18\%), seventh (22\%), or eighth (20\%) decade of life. The median age of patients with CA vs. HA bacteremia was not significantly different ( 67.5 vs. 58 years). CA bacteremia rarely occurred in individuals younger than 50 years. Of the 281 patients with bacteremia, $164(58 \%)$ were male and 117 
Table 1. Prevalence of underlying conditions with regard to acquisition of bacteremia due to S. aureus.

\begin{tabular}{|c|c|c|c|c|}
\hline \multirow[b]{2}{*}{ Predisposing factor } & \multicolumn{3}{|c|}{$\begin{array}{l}\text { No. (\%) of patients with factor } \\
\text { per type of bacteremia }\end{array}$} & \multirow[b]{2}{*}{$P$ valuet } \\
\hline & $\begin{array}{c}\text { Total } \\
\left(n=281^{*}\right)\end{array}$ & $\begin{array}{c}\text { CA } \\
(n=80)\end{array}$ & $\begin{array}{c}\text { HA } \\
(n=200)\end{array}$ & \\
\hline Intravascular device & $160(57)$ & 0 & $160(80)$ & $<.001$ \\
\hline Surgical wound & $103(37)$ & $6(7.5)$ & $96(48)$ & $<.001$ \\
\hline Arteriosclerotic heart disease & $69(25)$ & $20(25)$ & $49(25)$ & NS \\
\hline Previous hospitalization & $61(22)$ & $9(11)$ & $51(26)$ & $<.005$ \\
\hline Implants ${ }^{\ddagger}$ & $53(19)$ & $11(14)$ & $42(21)$ & NS \\
\hline Alcohol abuse & $51(19)$ & $7(9)$ & $44(23)$ & .035 \\
\hline Malignancy & $50(18)$ & $11(14)$ & $39(20)$ & NS \\
\hline Corticosteroids & $49(17)$ & $5(6)$ & $44(22)$ & $<.007$ \\
\hline Diabetes mellitus & $48(17)$ & $18(23)$ & $30(15)$ & NS \\
\hline Trauma & $41(15)$ & $2(2)$ & $39(20)$ & $<.001$ \\
\hline Chronic renal failure & $34(12)$ & $11(14)$ & $23(11.5)$ & NS \\
\hline Cytostatic and immunosuppressive therapy & $29(10)$ & $4(5)$ & $25(12.5)$ & NS \\
\hline Chronic rheumatic heart disease & $23(8)$ & $10(12.5)$ & $13(6.5)$ & NS \\
\hline Tracheostomy/artificial ventilation & $19(7)$ & $\ldots$ & $19(9.5)$ & NS \\
\hline Cirrhosis of liver & $15(5)$ & $2(2.5)$ & $13(6.5)$ & NS \\
\hline Cardiomyopathy & $11(4)$ & $4(5)$ & $7(3.5)$ & NS \\
\hline Rheumatoid arthritis & $8(3)$ & $5(6)$ & $3(1.5)$ & NS \\
\hline Intravenous drug abuse & $4(1.4)$ & $3(4)$ & $1(0.5)$ & NS \\
\hline Congenital heart disease & $1(0.4)$ & $1(1)$ & $\ldots$ & NS \\
\hline Other & $43(15)$ & $11(14)$ & $32(16)$ & NS \\
\hline None & $13(5)$ & $9(11)$ & $4(2)$ & NS \\
\hline
\end{tabular}

(42\%) were female. CA bacteremia occurred more frequently in females (33\%) than in males $(25 \%)$.

Underlying conditions. Table 1 shows the underlying conditions that were present in 268 subjects (95\%). They were found in $98 \%$ of patients with HA bacteremia and $87 \%$ of patients with CA bacteremia. Many patients had more than one underlying condition. There were only four patients with a history of intravenous drug abuse. No patient was infected with the human immunodeficiency virus. For patients with HA bacteremia, predisposing factors more often were intravenous catheters, surgical wounds, previous hospitalization, alcohol abuse, therapy with corticosteroids, and trauma. Of the 200 patients with HA bacteremia, 160 (80\%) had an intravascular device; this device was proved bacteriologically and/or clinically to be the portal of entry for $S$. aureus for $38.5 \%$ of these patients (table 2).

Clinical signs and symptoms and laboratory findings. At the time their first positive blood culture results were obtained, $91 \%$ of the patients had fever and the general condition of $85 \%$ was impaired. Thirty-eight percent presented with clouding of consciousness, and one-quarter suffered from chills. Vomiting, nausea, myalgia, and dyspnea were reported in $13 \%-14 \%$. Shock (9\%), hemiplegia (6\%), stiff neck $(4 \%)$, diarrhea $(2.5 \%)$, headache (1.5\%), and seizures (1\%) were less frequent. Only $1 \%$ showed no clinical abnormalities at the time the hemoculture was performed.

Primary vs. secondary bacteremia. In 219 patients (78\%) an apparent primary site of $S$. aureus infection was identified. Among patients with HA bacteremia the detection of a portal of entry (i.e., secondary bacteremia) was more common than among patients with CA bacteremia ( $88 \%$ vs. $54 \% ; \chi^{2}=$ $37.355 ; P<.001)$. The sites of primary foci are summarized in table 2.

Complicated vs. uncomplicated bacteremia. Among the whole study group, 176 patients $(63 \%)$ had complicated bacteremia, as defined by the existence of a nonremovable initial focus. Significantly fewer patients with CA bacteremia had a removable focus than did patients with HA bacteremia $\left(2.5 \%\right.$ vs. $\left.51 \% ; \chi^{2}=51.810 ; P<.001\right)$.

Clinical significance of bacteremia. Overall, $60 \%$ of the cases of bacteremia were clinically significant. In $4 \%$ no classification could be made. Sixty-eight percent of cases of HA bacteremia but only $46 \%$ of cases of CA bacteremia were clinically significant $\left(\chi^{2}=9.874 ; P<.002\right)$. 
Table 2. Portal of entry for S. aureus.

\begin{tabular}{lcc}
\hline & $\begin{array}{c}\text { No. of patients (\%) with indicated } \\
\text { type of acquisition of infection }\end{array}$ \\
\cline { 2 - 3 } & $\begin{array}{c}\text { CA } \\
\text { Site or condition }\end{array}$ & HA \\
\hline Peripheral iv catheter & $\ldots$ & $(n=200)$ \\
Central iv catheter & $\ldots$ & $24(12)$ \\
Wounds & $\ldots$ & $53(26.5)$ \\
Primary pneumonia & $6(7.5)$ & $29(14.5)$ \\
Urinary catheter & $3(4)$ & $21(10.5)$ \\
Injections & $1(1)$ & $13(6.5)$ \\
Implants & $4(5)$ & $1(0.5)$ \\
Skin & $6(7.5)$ & $7(3.5)$ \\
Others* & $23(29)$ & $5(2.5)$ \\
None & $37(46)$ & $23(11.5)$ \\
\hline
\end{tabular}

* Perforating ulcer of the foot and/or osteomyelitis (18), transvenous pacemaker (5), bronchitis (3), dental abscess (3), arteriovenous shunt infection (3), urinary tract infection (3), gluteal abscess (2), intraarticular injection (2), epidural catheter (1), vesical catheter (1), pleural catheter (1), acne conglobata (1), intestinal infection (1), throat infection (1), and anterior and posterior nasal tamponade (I).

Secondary foci of bacteremia. Metastatic staphylococcal foci demonstrated by roentgenography, scintigraphy, surgical drainage, puncture, histology, or autopsy were noted in 75 patients (27\%). The foci were in the following sites: joint (27 patients), kidney (22), CNS (21), myocardium (17), skin (12), intervertebral disk (11), lung (10), bone (8), spleen (7), subcutis (3), liver (3), vascular system (3), hematomas (3), small intestine (2), and the eye, bursa olecrani, pericardium, pancreas, and thyroid gland ( 1 each). In most cases (50.7\%) a single metastatic focus was detected. Metastasizing foci were more common in cases of primary vs. secondary bacteremia ( $51 \%$ vs. $\left.21 \% ; \chi^{2}=21.471 ; P<.001\right)$. They were also more frequent in cases of CA bacteremia than in those of HA bacteremia ( $43 \%$ vs. $21 \% ; \chi^{2}=14.036 ; P<.001$ ). Only $19 \%$ of cases of uncomplicated bacteremia were followed by the development of secondary foci, as compared with $32 \%$ of cases of complicated bacteremia $\left(\chi^{2}=4.949, P<.05\right)$.

Endocarditis. Endocarditis was diagnosed in $8.2 \%$ of all patients with bacteremia at any time during the course of hospitalization. In spite of the various types of bacteremia, only the presence or absence of primary foci correlated with a significant difference in the incidence of endocarditis $6 \%$ vs. $\left.17 \% ; \chi^{2}=4.9128 ; P=.0267\right)$. Fourteen of the patients with CA bacteremia but only $6 \%$ with HA bacteremia developed endocarditis $(P=.09)$.

Acute complications. Septic shock was observed in 48 patients $(17.1 \%)$; acute respiratory distress syndrome, in 14 (5\%); and disseminated intravascular coagulation, in 28 $(10 \%)$. The latter complication was more common in cases of CA vs. HA bacteremia $\left(17.5 \%\right.$ vs. $7 \% ; \chi^{2}=4.5714 ; P$ $=.0325)$.
Prognosis. The large proportion of patients with a poor prognosis (57\%) points to the importance of the underlying condition as risk factor for $S$. aureus bacteremia. Patients with HA bacteremia had a poor prognosis more frequently than did patients with CA bacteremia $\left(63.5 \%\right.$ vs. $41 \% ; \chi^{2}=$ 9.704; $P=.002$ ). Similarly, a good prognosis was more frequent among patients with CA bacteremia (15\%) than among those with HA bacteremia $(4 \%)\left(\chi^{2}=5.8156 ; P\right.$ $=.016)$.

Therapy. Only $43 \%$ of all patients with bacteremia could be considered cured, and in $14 \%$ an improvement in their condition was noted. Sustained treatment failure or relapse was observed in $24 \%$ and $8 \%$ of the patients, respectively. In $11 \%$ of cases no clear statement about the efficacy of therapy could be made, either because antibiotics were withheld owing to an unfavorable prognosis in regard to underlying disease or because the patient was transferred to another hospital. A significantly higher percentage of patients with HA bacteremia were cured than were patients with CA bacteremia ( $47 \%$ vs. $\left.31 \% ; \chi^{2}=4.728 ; P=.029\right)$. Initially over one-quarter of all patients with bacteremia received inappropriate antibiotic treatment. For uncomplicated cases of bacteremia, $17 \%$ of the initial treatments were inappropriate, as compared with $32 \%$ for complicated cases of bacteremia $\left(\chi^{2}\right.$ $=6.834 ; P=.009$ ). Sixty-one patients with bacteremia underwent surgical removal of a primary or secondary focus. Surgery was more frequently performed in patients with complicated bacteremia than in those with uncomplicated bacteremia ( $29 \%$ vs. $9.5 \% ; \chi^{2}=14.742 ; P<.001$ ).

The median duration of antimicrobial therapy for 257 bacteremic patients was 15 days (range, $0-157$ days). Seventy patients with CA bacteremia received antibiotics for a median of 22 days (mean, 30.7 days), and 187 patients with nosocomial infection were treated for a median of 12 days (mean, 16.2 days). A total of 104 patients with uncomplicated bacteremia were treated with use of an intravenous catheter for a median of 9.5 days (mean, 11.3 days; range, $0-60$ days); two patients were cured by catheter removal without use of antibiotics.

At the time of the first blood culture positive for $S$. aureus, the treating physician judged 48 (19\%) of 247 patients to be irrelevantly infected or the blood culture to be contaminated. For these patients no therapy was started, or an inappropriate initial treatment was not changed according to the susceptibility pattern of the isolate. After the introduction of an infectious disease service at the hospital in 1982, the number of misinterpreted blood cultures dropped from 12 to 4.8 per year.

Mortality rate. The overall mortality rate among patients with $S$. aureus bacteremia was $33.6 \%$. In $20 \%$ of cases, death was directly related to $S$. aureus, and in $28 \%$, to the underlying illness. In $52 \%$ of the patients, the cause was unclear or not due to the $S$. aureus infection alone. A comparison of the 
death rates associated with differently defined bacteremia demonstrated that uncomplicated bacteremia was associated with a lower mortality rate than was complicated bacteremia ( $24 \%$ vs. $40 \% ; \chi^{2}=6.829, P<.01$ ). Similarly, secondary bacteremia was also associated with a lower mortality rate ( $30 \%$ vs. $45 \%, \chi^{2}=4.183, P=.05$ ). There was no significance in the differences between CA and HA bacteremia and between clinically significant and insignificant bacteremia in regard to mortality. The mortality rate was higher among patients with endocarditis $(65 \% ; n=23)$ as compared with patients with secondary foci of infection who did not have endocarditis $(49 \%, n=57)\left(\chi^{2}=4.5859 ; P=.03\right)$. The mortality rate among patients without any metastatic infections $(n=201)$ was $25 \%$, a significantly lower rate than the $49 \%$ found among patients with secondary foci $\left(\chi^{2}=\right.$ $30.7272 ; P<.001)$.

Sensitivity pattern. During the investigated period, only one methicillin-resistant strain-which was also resistant to erythromycin, doxycycline, chloramphenicol, and trimethoprim-sulfamethoxazole-was isolated.

\section{Discussion}

The aim of our study was to evaluate different previously published clinical case definitions of $S$. aureus bacteremia $[17,23,32-36]$. $S$. aureus remains a significant pathogen, causing $13.6 \%$ of all cases of bacteremia at our hospital. This finding is consistent with the results of other series showing rates of 7.5\%-25\% (mostly between $10 \%$ and $15 \%$ ) $[1,7,9$, $10,12,13,16-18,20,36,38-42]$. In the literature, varied meanings of the same terms regarding the different clinical case definitions can be found. Secondary bacteremia, most often defined as bacteremia with an identified portal of entry, has been described also as bacteremia developing during the course of another fatal disease [8]. Complicated bacteremia has been described as bacteremia complicated by secondary foci and other clinical findings [34] as well as bacteremia with an undefined or nonremovable focus [32, 33]. Furthermore, cases of HA bacteremia have been defined differently with use of various endpoints (48 hours [10, 16-18, $34,43], 72$ hours [35], 96 hours [9, 23, 44], or even 1 week [22] after admission to the hospital) for distinguishing them from cases of CA bacteremia. These variations impede a reliable comparison.

In our series the comparison of various clinical case definitions thought to be predictive of the outcome $[9,10,15,21$, $23,28,45-48]$ shows that a statistically significant better outcome with regard to mortality rate, occurrence of secondary foci, and endocarditis is achieved only in cases of secondary bacteremia. The higher incidence of endocarditis and secondary foci in cases of primary bacteremia, which was also observed by others $[5,23,24]$, may be due to delayed treat- ment with effective antibiotics in the absence of a primary focus.

HA bacteremia was less often followed by secondary foci and endocarditis but was associated with a mortality rate similar to that associated with CA bacteremia, a finding which was also observed in a newer prospective study [49]. The more benign course of HA bacteremia is well known from the literature $[9,48,50,51]$. However, the mortality rate remains high, and additional factors such as underlying conditions [24, 36, 43], the age of the patients [10, 21, 23, 45], and the nature of the primary infection appear to influence the outcome. Cases of uncomplicated bacteremia were significantly less often followed by secondary foci and were associated with a lower mortality rate than were complicated cases, but the two did not differ in terms of the associated incidence rate of endocarditis. A low risk of subsequent secondary foci after an episode of uncomplicated bacteremia was also reported in two prospective studies [52. 53], whereas the low incidence of endocarditis in these and other investigations $[9,41]$ contrasted with the results of our study. Watanakunakorn and Baird [46] mentioned in their study the significant risk of developing endocarditis after an episode of uncomplicated bacteremia, whereas in a recent publication [40] the risk was called "small but definite."

In our series, the mortality rate was highest among patients with no or nonremovable foci, a finding which may be explained by the delay in instituting effective treatment. The earlier awareness of the possibility of bacteremia in cases with obvious local staphylococcal lesions and, consequently, the institution of significantly more appropriate and earlier treatment may have prevented many sequelae. Michel et al. [17] showed a 4.4-fold higher mortality rate among patients with "clinically significant bacteremia," as defined in their Methods section. In contrast, in our series this classification was not discriminative in terms of the fatality rate. This may be because of the fact that before the introduction of an infectious disease service, a considerable proportion of patients with subtle symptoms received no or inadequate therapy in our hospital.

In conclusion, $S$. aureus bacteremia remains a major problem and is associated with a high rate of mortality. The presence of a source of bacteremia was the only factor predictive of the outcome. Patients for whom the source of bacteremia was defined had a better prognosis with regard to mortality rate, incidence of endocarditis, and secondary foci. Our data suggest that an improvement of the prognosis could be achieved by more competent interpretation of positive blood cultures yielding $S$. aureus and by earlier initiation of antistaphylococcal therapy.

\section{References}

1. Maki DG. Nosocomial bacteremia. An epidemiologic overview. Am J Med 1981;70:719-32. 
2. Kreger BE, Craven DE, Carling PC. McCabe WR. Gram-negative bacteremia. III. Reassessment of etiology, epidemiology and ecology in 612 patients. Am J Med 1980;68:332-43.

3. Finland M. Jones WF. Barnes MW. Council on drugs. Occurrence of serious bacterial infections since introduction of antibacterial agents. JAMA 1959;170:2188-97.

4. Faber V, Jessen O, Rosendal K, Eriksen KR. Staphylococcal bacteremia. Clinical and bacteriological observations in 201 cases. BMJ 1960;1832-6.

5. Sheagren JN. Staphylococcus aureus. The persistent pathogen (first of two parts). N Engl J Med 1984;310:1368-73.

6. Allen JR. Hightower AW, Martin SM, Dixon RE. Secular trends in nosocomial infections: 1970-1979. Am J Med 1981;70: 389-92.

7. Lacey RW, Keyworth N, Lincoln C. Staphylococci in the U.K.: a review. J Antimicrob Chemother 1984;14(suppl D):19-25.

8. Smith IM, Vickers AB. Natural history of 338 treated and untreated patients with staphylococcal septicaemia (1936-1955). Lancet 1960;1:1318-22.

9. Finkelstein R, Sobel JD, Nagler A, Merzbach D. Staphylococcus aureus bacteremia and endocarditis: comparison of nosocomial and community-acquired infection. J Med 1984;15:193-211.

10. Shah M, Watanakunakorn C. Changing patterns of Staphylococcus aureus bacteremia. Am J Med Sci 1979;278:115-21.

11. Scheckler WE. Septicemia in a community hospital 1970 through 1973. JAMA 1977;237:1938-41.

12. McGowan JE Jr. Septicaemia: changing patterns of causative organisms and underlying conditions. In: Shanson DC, ed. Septicaemia and endocarditis. Oxford: Oxford University Press, 1989:5-48.

13. Flournoy DJ, Adkins L. Understanding the blood culture report. Am J Infect Control 1986;14:41-6.

14. MacGregor RR, Beaty HN. Evaluation of positive blood cultures. Guidelines for early differentiation of contaminated from valid positive cultures. Arch Intern Med 1972;130:84-7.

15. Michel MF, Priem CC. Positive blood cultures in a university hospital in the Netherlands. Infection 1981;9:283-9.

16. Weinstein MP, Reller LB, Murphy JR, Lichtenstein KA. The clinical significance of positive blood cultures: a comprehensive analysis of 500 episodes of bacteremia and fungemia in adults. 1. Laboratory and epidemiologic observations. Rev Infect Dis 1983;5:35-53.

17. Michel MF. Priem CC, Verbrugh HA, Goessens WHF. Staphylococcus aureus bacteremia in a Dutch teaching hospital. Infection 1985; 13:267-72.

18. McGowan JE Jr, Barnes MW, Finland M. Bacteremia at Boston City Hospital: occurrence and mortality during 12 selected years (19351972), with special reference to hospital-acquired cases. J Infect Dis 1975;132:316-35.

19. Fauci AS, Dale DC, Balow JE. Glucocorticosteroid therapy: mechanisms of action and clinical considerations. Ann Intern Med 1976;84:304-15.

20. Weinstein MP, Murphy JR, Reller LB, Lichtenstein KA. The clinical significance of positive blood cultures: a comprehensive analysis of 500 episodes of bacteremia and fungemia in adults. II. Clinical observations, with special reference to factors influencing prognosis. Rev Infect Dis 1983;5:54-70.

21. Julander I. Unfavourable prognostic factors in Staphylococcus aureus septicemia and endocarditis. Scand J Infect Dis 1985;17:179-87.

22. Kilton LJ, Fossieck BE, Cohen MH, Parker RH. Bacteremia due to gram-positive cocci in patients with neoplastic disease. Am J Med 1979;66:596-602.

23. Nolan CM, Beaty HN. Staphylococcus aureus bacteremia. Current clinical patterns. Am J Med 1976;60:495-500.
24. Cluff LE, Renolds RC, Page DL, Breckenridge JL. Staphylococcal bacteremia and altered host resistance. Ann Intern Med 1968;69:85973.

25. McNeill KM, Strong JE, Lockwood WR. Bacterial endocarditis: an analysis of factors affecting long-term survival. Am Heart J 1978;95:448-53.

26. Musher DM, McKenzie SO. Infections due to Staphylococcus aureus. Medicine (Baltimore) 1977;56:383-409.

27. Dobkin JF, Miller MH, Steigbigel NH. Septicemia in patients on chronic hemodialysis. Ann Intern Med 1978;88:28-33.

28. Nicholls A, Edward N, Catto GRD. Staphylococcal septicaemia, endocarditis, and osteomyelitis in dialysis and renal transplant patients. Postgrad Med J 1980;56:642-8.

29. Plaut ME, Palaszynski F, Bjornsson S, Yates JW, Henderson ES. Staphylococcal bacteremia in a "germ-free" unit. Arch Intern Med 1976;136:1238-40.

30. Hanifin JM, Homburger HA. Staphylococcal colonization, infection, and atopic dermatitis-association not etiology. $\mathbf{J}$ Allergy Clin Immunol 1986;78:563-66.

31. Scheidegger $C, Z$ Zimmerli $W$. Infectious complications in drug addicts: seven-year review of 269 hospitalized narcotics abusers in Switzerland. Rev Infect Dis 1989;1 1:486-93.

32. Iannini PB, Crossley K. Therapy of Staphylococcus aureus bacteremia associated with a removable focus of infection. Ann Intern Med 1976;84:558-60.

33. Verbrugh HA, Peters R, Goessens WHF, Michel MF. Distinguishing complicated from uncomplicated bacteremia caused by Staphylococcus aureus: the value of "new" and "old" serological tests. J Infect Dis 1986;153:109-15.

34. Libman H, Arbeit RD. Complications associated with Staphylococcus aureus bacteremia. Arch Intern Med 1984;144:541-5.

35. Bayer AS, Lam K, Ginzton L, Norman DC, Chiu CY, Ward JI. Staphylococcus aureus bacteremia. Clinical, serologic, and echocardiographic findings in patients with and without endocarditis. Arch Intern Med 1987;147:457-62.

36. Gransden WR, Eykyn SJ, Phillips I. Staphylococcus aureus bacteremia. BMJ 1984;288:300-3.

37. Waldvogel FA. Staphylococcus aureus (including toxic shock syndrome). In: Mandell GL, Douglas RG Jr, Bennett JE, eds. Principles and practice of infectious diseases. 3rd ed. New York: John Wiley \& Sons, 1990:1489-510

38. Ljungman P, Malmborg AS, Nystrom B, Tillegard A. Bacteremia in a Swedish university hospital: a one-year prospective study in 1981 and a comparison with 1975-76. Infection 1984;12:243-7.

39. McGowan JE Jr, Parrott PL, Duty VP. Nosocomial bacteremia. JAMA 1977;237:2727-9.

40. Eykyn SJ, Gransden WR, Phillips I. The causative organisms of septicaemia and their epidemiology. J Antimicrob Chemother 1990; 25(suppl C):41-58.

41. Ispahani P, Pearson NJ, Greenwood D. An analysis of community and hospital-acquired bacteremia in a large teaching hospital in the United Kingdom. Q J Med 1987;241:427-40.

42. Julander I. Septicemia and endocarditis, 1965-1980, in a Swedish university hospital for infectious diseases. Infection 1987;15:177-83.

43. Setia U, Gross PA. Bacteremia in a community hospital. Arch Intern Med 1977;137:1698-701.

44. Mirimanoff RO, Glauser MP. Endocarditis during Staphylococcus aureus septicemia in a population of non-drug addicts. Arch Intern Med 1982;142:1311-3.

45. Espersen F, Frimodt-Moller N. Staphylococcus aureus endocarditis. A review of 119 cases. Arch Intern Med 1986;146:1118-21. 
46. Watanakunakorn C, Baird IM. Staphylococcus aureus bacteremia and endocarditis associated with a removable infected intravenous device. Am J Med 1977;63:253-6.

47. Bayer AS. Staphylococcal bacteremia and endocarditis. State of the art. Arch Intern Med 1982;142:1169-77.

48. Eykyn SJ. Staphylococcal sepsis. The changing pattern of disease and therapy. Lancet 1988;1:100-3.

49. Mylotte JM, McDermott C, Spooner JA. Prospective study of 114 consecutive episodes of Staphylococcus aureus bacteremia. Rev Infect Dis 1987;9:891-907.
50. Austin TW, Wallace JF. Staphylococcus aureus bacteremia: a critical review of its treatment and association with infective endocarditis. Infection 1973:1:214-7.

51. Hedström SA, Christensson B. Staphylococcus aureus septicaemia and endocarditis at the university hospital in Lund 1976-1980. Scand J Infect Dis Suppl 1983;41:38-46.

52. Mylotte JM, McDermott C. Staphylococcus aureus bacteremia caused by infected intravenous catheters. Am J Infect Control 1987;1 5:1-6.

53. Ehni WF, Reller LB. Short course therapy for catheter-associated Staphylococcus aureus bacteremia. Arch Intern Med 1989;149:533-6. 